\title{
Conditioned medium from tonsil-derived mesenchymal stem cells promotes adiponectin production
}

\author{
YU-HEE KIM ${ }^{1}$, KYUNG-AH CHO ${ }^{1}$, MINHWA PARK ${ }^{1}$, \\ JULIE A. WEBSTER ${ }^{2}$, SO-YOUN WOO ${ }^{1}$ and KYUNG-HA RYU ${ }^{3}$ \\ ${ }^{1}$ Department of Microbiology, School of Medicine, Ewha Womans University, Seoul 07985, Republic of Korea; \\ ${ }^{2}$ School of Biomedical Sciences, University of Queensland, Brisbane, QLD 4072, Australia; ${ }^{3}$ Department of \\ Pediatrics, School of Medicine, Ewha Womans University, Seoul 07985, Republic of Korea
}

Received March 9, 2017; Accepted June 15, 2017

DOI: $10.3892 / \mathrm{mmr} .2017 .7335$

\begin{abstract}
Mesenchymal stem cells (MSCs) are often considered to be a good source for the development of regenerative medicine. Previously, we reported that tonsil-derived MSC conditioned medium (T-MSC CM) produces visceral fat reducing effects. As reduced visceral adiposity is closely associated with an increase in circulating adiponectin, the present study investigated the effects of T-MSC CM on adiponectin production. T-MSC CM was collected from previously isolated and characterized T-MSCs and injected into senescence-accelerated mouse prone 6 mice, which exhibit characteristics of aging and obesity. The results demonstrated a reduction in mouse weight and epididymal adipose tissue (eAT) mass following injection of T-MSC CM. Significant increases in adiponectin expression in the eAT, and total and high molecular weight (HMW) adiponectin in the circulation were observed in the T-MSC CM-injected mice compared with control mice using reverse transcription-quantitative polymerase chain reaction, western blot analysis and ELISA. In 3T3-L1 adipocytes, T-MSC CM treatment increased adiponectin secretion and multimerization, as detected using western blotting under non-reducing and non-heat-denaturing conditions. Furthermore, glucose oxidase was used to induce oxidative stress in 3T3-L1 adipocytes and it was observed that T-MSC CM reduced reactive oxygen species production and the expression of certain oxidative stress markers. In addition, the results also demonstrated that the production of HMW adiponectin was increased, which indicates that T-MSC CM may enhance adiponectin multimerization via amelioration of oxidative stress. Further studies are required to elucidate
\end{abstract}

Correspondence to: Professor Kyung-Ha Ryu, Department of Pediatrics, School of Medicine, Ewha Womans University, 1071 Anyangcheon-ro, Yangcheon-Gu, Seoul 07985, Republic of Korea

E-mail: ykh@ewha.ac.kr

Key words: high molecular weight adiponectin, tonsil-derived mesenchymal stem cells, adiponectin, oxidative stress, 3T3-L1 adipocyte, senescence-accelerated mouse prone 6 anti-oxidant molecules secreted from T-MSCs, and these results highlight the potential therapeutic relevance of T-MSC $\mathrm{CM}$ for the treatment of obesity or obesity-associated diseases.

\section{Introduction}

Mesenchymal stem cells (MSCs) are often considered to be a good source for the development of regenerative medicine due to the self-renewal and multipotent properties of these cells. The MSC secretome is another area of research that will ultimately contribute to the development of cell-free therapy $(1,2)$. From soluble factors to extracellular vesicles, MSCs produce a variety of biologically active secreted proteins. As only a small percentage of transplanted MSCs are successfully engrafted, differentiated and functional in the target tissue $(3,4)$, it was suggested that endocrine and immunomodulatory effects of the MSC secretome may have important roles in tissue regeneration $(5,6)$.

MSCs can be obtained from various tissues, including the bone marrow, adipose tissue, placenta and umbilical cord. In 2008, human tonsils were described as a novel source of MSCs and these MSCs are termed tonsil-derived MSCs (T-MSCs) (7). The characteristics and potential of MSCs to be used for cell banking have been previously reported (8). The therapeutic potential of T-MSCs in the treatment of liver $(9,10)$, pancreas (11), parathyroid gland (12), skeletal muscle (13), and bone (14) diseases, as well as the mechanisms that underlie their immunomodulatory effects (15-18), have been described previously.

Adiponectin, which is primarily produced and secreted by adipocytes, evokes several beneficial metabolic functions via insulin-sensitizing, anti-inflammatory and cardiovascular protective effects (19-21). Although adiponectin is secreted from adipocytes, the levels of this protein in circulation are not always correlated with body mass index (BMI). Instead, weight reduction paradoxically increases adiponectin in circulation (22,23). Adiponectin circulates in different multimer forms: Low molecular weight form (LMW; trimer), medium molecular weight form (hexamer) and high molecular weight form (HMW; octadecamer or higher). LMW and HMW adiponectin promote glucose and lipid metabolism in hepatocytes, while LMW primarily acts on myocytes $(19,21)$. 
Thus, promotion of adiponectin multimerization rather than an increase and/or decrease of total adiponectin affects the function of adiponectin.

In a previous study, we observed that T-MSC CM injection reduces the body weight of senescence-accelerated mouse prone 6 (SAMP6) mice (14). In addition, a selective decrease in visceral adiposity was observed in the previous study, and we also reported that T-MSC CM has the potential to inhibit adipogenesis in vitro. Given that weight reduction is closely associated with the improvement of whole body metabolism due, in part, to increased levels of adiponectin in circulation, the present study aimed to determine whether T-MSC CM regulates adiponectin expression, secretion, and/or multimerization in SAMP6 aging mice and 3T3-L1 adipocytes.

\section{Materials and methods}

T-MSC culture and T-MSC CM preparation. Previously isolated and characterized human T-MSCs $(8,24)$ were cultured in Dulbecco's modified Eagle's medium (DMEM) high-glucose medium (Welgene, Inc., Gyeongsan, Korea) supplemented with $10 \%$ fetal bovine serum (FBS; Welgene, Inc.), $100 \mathrm{IU} / \mathrm{ml}$ penicillin and $100 \mu \mathrm{g} / \mathrm{ml}$ streptomycin (Welgene, Inc.). Cells were maintained at $37^{\circ} \mathrm{C}$ in a humidified $5 \% \mathrm{CO}_{2}$ atmosphere. When cells reached $80 \%$ confluency, fresh culture medium was added and the cells were incubated for an additional $48 \mathrm{~h}$. T-MSC CM was harvested and concentrated 10-fold using 3-kDa cut-off Amicon Ultra centrifugal filter units (Merck KGaA, Darmstadt, Germany) at a speed of $3,800 \mathrm{x} \mathrm{g}$ for $30 \mathrm{~min}$ at room temperature.

Animals. A total of 12 male SAMP6 mice (age, 4 months; weight, $47.04 \pm 2.87 \mathrm{~g}$ ) were purchased from Japan SLC, Inc. (Hamamatsu, Japan) and maintained at $21-23^{\circ} \mathrm{C}$ with $51-54 \%$ humidity and a 12-h light/dark cycle under conventional conditions, with food and water supplied ad libitum. At 7 months of age (weight, $52.64 \pm 4.66 \mathrm{~g}$ ), mice were divided into two groups ( $\mathrm{n}=6$ mice/group) and injected via tail vein with either culture medium alone (control) or culture supernatant that was collected and concentrated after a $48 \mathrm{~h}$ incubation of $0.8 \times 10^{6} \mathrm{~T}-\mathrm{MSC}$ (T-MSC CM). Treatments were performed twice a week for 2 weeks using freshly prepared CM. Mice were sacrificed at 9 months of age by cervical dislocation, and whole blood and organs were harvested for further analysis. Experiments and procedures were approved by the Animal Ethics Committee at Ewha Womans University School of Medicine (Seoul, Korea; no. ESM 14-0278), and all experiments were performed in accordance with relevant guidelines and regulations.

Hematoxylin and eosin (H\&E) staining. Mouse epididymal adipose tissue (eAT) was isolated and fixed with a $4 \%$ paraformaldehyde solution overnight at $4^{\circ} \mathrm{C}$. Paraffin-embedded eAT was sectioned at $4 \mu \mathrm{m}$ and subjected to H\&E staining. Briefly, cells were stained with $0.7 \%$ hematoxylin for $2 \mathrm{~min}$ and then with $5 \%$ eosin for $1.5 \mathrm{~min}$ at room temperature. Stained sections were observed using an Olympus BX50 light microscope (Olympus Corporation, Tokyo, Japan) and images were captured under x100 magnification.
Adiponectin ELISA. Blood samples ( 200 $\mu \mathrm{l})$ were collected in a tube containing heparin sodium (JW Pharmaceutical, Seoul, Korea) from the facial vein of 7- and 9-month-old mice following $6 \mathrm{~h}$ of fasting. Plasma proteins were separated by centrifugation at $1,000 \mathrm{x} \mathrm{g}$ for $10 \mathrm{~min}$ at room temperature. The adiponectin concentration was measured using the Adiponectin Total, HMW ELISA kit (cat. no. 47-ADPMS-E01; Alpco Diagnostics, Salem, NH, USA), according to the manufacturer's protocol.

Cell culture. The mouse preadipocyte cell line 3T3-L1 was purchased from the Korean Cell Line Bank (Seoul, Korea) and maintained in DMEM high-glucose medium supplemented with $10 \% \mathrm{FBS}, 100 \mathrm{IU} / \mathrm{ml}$ penicillin, and $100 \mu \mathrm{g} / \mathrm{ml}$ streptomycin. After reaching $100 \%$ confluency, the medium was changed and cells were maintained for an additional 3 days. Cells were subsequently induced to differentiate to adipocytes (day 0). To induce adipocyte differentiation, high-glucose DMEM containing 10\% FBS, $100 \mathrm{IU} / \mathrm{ml}$ penicillin, and $100 \mu \mathrm{g} / \mathrm{ml}$ streptomycin supplemented with $2 \mathrm{mg} / \mathrm{ml}$ insulin, $0.25 \mu \mathrm{M}$ dexamethasone and $0.5 \mathrm{mM} 3$-isobutyl-1-methylxanthine (all from Sigma-Aldrich; Merck KGaA) was used. On day 3 , the medium was replaced with medium containing insulin only and cells were cultured for another 4 days at $37^{\circ} \mathrm{C}$ in a humidified $5 \% \mathrm{CO}_{2}$ atmosphere. Concentrated culture medium or T-MSC CM was added between days 7 and 10 . On day 10, the cells were rinsed with PBS and incubated in serum-free medium overnight. Culture supernatants and cells were harvested the next day for further analyses. To induce oxidative stress in mature adipocytes, cells were pretreated with $50 \mathrm{mU} / \mathrm{ml}$ glucose oxidase (Sigma-Aldrich; Merck $\mathrm{KGaA}$ ) for $2 \mathrm{~h}$, followed by overnight treatment with T-MSC $\mathrm{CM}$ at $37^{\circ} \mathrm{C}$.

$R N A$ extraction and reverse transcription $(R T)$. To harvest adipocytes, the eAT was weighed and finely minced. Minced tissue was transferred to a 4-times greater volume of Hank's Balanced Salt Solution buffer (Welgene, Inc.) containing $1 \mathrm{mg} / \mathrm{ml}$ collagenase I (Sigma-Aldrich; Merck KGaA) and $0.28 \mathrm{M}$ fatty acid-free bovine serum albumin (BSA; Sigma-Aldrich; Merck KGaA). Tissue digestion was performed by incubating the tissue sample for $2 \mathrm{~h}$ at $37^{\circ} \mathrm{C}$ in a shaking incubator. Digested tissue was filtered through a cell strainer and centrifuged at $100 \mathrm{x}$ g for $15 \mathrm{~min}$ at room temperature. The floating adipocyte fraction was transferred to a new tube for RNA extraction.

To extract RNA from mouse eAT or 3T3-L1 adipocytes $\left(9.5 \times 10^{5}\right)$, TRIzol reagent (Invitrogen; Thermo Fisher Scientific, Inc., Waltham, MA, USA) was used. Tissue homogenization was performed using a TissueRuptor (Qiagen GmbH, Hilden, Germany), and following phase separation by centrifugation at $12,000 \mathrm{x} \mathrm{g}$ for $15 \mathrm{~min}$ at $4^{\circ} \mathrm{C}$, RNA was isolated using NucleoSpin ${ }^{\circledR}$ RNA Clean-up kit (Macherey-Nagel GmbH \& Co. KG, Düren, Germany), according to the manufacturer's protocol. After determination of the concentration and purity of the RNA samples using BioPhotometer D30 (Eppendorf, Hamburg, Germany), $1 \mu \mathrm{g}$ RNA was used for cDNA synthesis using a ReverTra Ace- $\alpha$-kit (Toyobo Co., Ltd., Osaka, Japan). Reverse transcription was performed by incubation at $30^{\circ} \mathrm{C}$ for $10 \mathrm{~min}$, at $42^{\circ} \mathrm{C}$ for $20 \mathrm{~min}$ and at $99^{\circ} \mathrm{C}$ for $5 \mathrm{~min}$. 
$R T$-quantitative polymerase chain reaction ( $q P C R)$. To determine the expression of target genes, a primer pair $(0.4 \mu \mathrm{M})$ and SYBR-Green Real-time PCR Maser Mix (Toyobo Co., Ltd.) were mixed with the prepared cDNA. The primer sequences are listed in Table I. Amplification was performed in duplicate by 40 cycles of $15 \mathrm{sec}$ denaturation step at $95^{\circ} \mathrm{C}$ and a 1 min amplification and signal acquisition step at $60^{\circ} \mathrm{C}$ using StepOnePlus Real-Time PCR System (Applied Biosystems; Thermo Fisher Scientific, Inc.). Cycle threshold (Cq) values were obtained, and the relative expression level of a target gene was determined as 2 (cyclophilin Cq-target gene Cq) (25).

Western blot analysis. Whole protein lysates were isolated from mouse eAT or 3T3-L1 adipocytes $\left(9.5 \times 10^{5}\right)$ using PRO-PREP Protein Extraction Solution (Intron Biotechnology, Inc., Seongnam, Korea). Protein concentrations were determined using the Bradford assay with the Bio-Rad Protein assay solution (Bio-Rad Laboratories, Inc., Hercules, CA, USA). Samples ( $4 \mu \mathrm{g}$ for tissue lysates and $10 \mu \mathrm{g}$ for cell lysates) were resolved by $10 \%$ SDS-PAGE and transferred to Immobilon-P polyvinylidene fluoride membranes (EMD Millipore, Billerica, MA, USA). Membranes were blocked with 5\% skim milk in TBS containing 0.1\% Tween-20 (TBS-T) solution for $1 \mathrm{~h}$ at room temperature and were subsequently incubated with primary antibodies overnight at $4^{\circ} \mathrm{C}$. The following primary antibodies were used: Adiponectin (1:1,000, diluted in 5\% skim milk containing TBST; cat. no. ab25891; rabbit; Abcam, Cambridge, UK); and $\beta$-actin [1:2,000, diluted in 2\% BSA (Bovogen Biologicals, Pty, Ltd., East Keilor, Victoria, Australia) containing TBST; cat. no. A1978; mouse; Sigma-Aldrich; Merck KGaA]. The membranes were washed 3 times for $10 \mathrm{~min}$ in TBST and incubated with anti-rabbit (cat. no. BR170-6515; Bio-Rad Laboratories, Inc.) or anti-mouse (cat. no. BR170-6516; Bio-Rad Laboratories, Inc.) horseradish peroxidase-conjugated secondary antibodies (1:3,000, diluted in TBST) for $1 \mathrm{~h}$ at room temperature. Following incubation, membranes were washed 3 times for $10 \mathrm{~min}$ in TBST and developed using SuperSignal West Femto Maximum Sensitivity Substrate (Pierce; Thermo Fisher Scientific, Inc.). Images were obtained using ImageQuant LAS 4000 (GE Healthcare Life Sciences, Little Chalfont, UK).

To detect secreted adiponectin in cell culture medium, conditioned medium was prepared by incubating differentiated 3T3-L1 adipocytes $\left(3.8 \times 10^{5}\right)$ in serum-free DMEM $(500 \mu \mathrm{l})$ for $18 \mathrm{~h}$. Conditioned medium was concentrated 10 -fold using 3-kDa cut-off Amicon Ultra centrifugal filter units (Merck $\mathrm{KGaA}$ ) and centrifugation at $14,000 \mathrm{x} \mathrm{g}$ for $15 \mathrm{~min}$ at room temperature. Concentrated medium $(50 \mu \mathrm{l})$ was prepared in sample buffer $(0.5 \mathrm{M}$ Tris $/ \mathrm{HCl} \mathrm{pH} 6.8,25 \%$ glycerol, $10 \%$ SDS, 500 mM DTT, $1 \%$ bromophenol blue; Sigma-Aldrich; Merck KGaA) and $12 \mu \mathrm{l}$ samples were separated on $10 \%$ SDS-PAGE for the detection of total secreted adiponectin. To detect adiponectin in multimer forms, concentrated medium was prepared in non-reducing and non-heat-denaturing conditions using sample buffer lacking reducing agents, and $12 \mu \mathrm{l}$ samples were subsequently separated on $4-15 \%$ precast polyacrylamide gradient gels (Bio-Rad Laboratories, Inc.). Blots were semi-quantified by densitometric analysis using UN-SCAN-IT gel analysis software version 6.1 (Silk Scientific, Inc., Orem, UT, USA).
Reactive oxygen species (ROS) and reactive nitrogen species (RNS) measurement. ROS and RNS production in total cell lysates or culture medium was measured using OxiSelect ${ }^{\mathrm{TM}}$ In Vitro ROS/RNS assay kit (Cell Biolabs, Inc., San Diego, CA, USA), according to the manufacturer's protocol. Cellular ROS/RNS levels were normalized to total protein measured by the BCA Protein assay kit (Pierce; Thermo Fisher Scientific, Inc.).

Statistical analysis. Student's t-test or one-way analysis of variance with Dunnett's multiple comparisons test was performed for statistical analysis using GraphPad Prism 5.0 (GraphPad Software, Inc., La Jolla, CA, USA). Data are presented as the mean \pm standard error of the mean from 3-4 independent experiments. $\mathrm{P}<0.05$ was considered to indicate a statistically significant difference.

\section{Results}

T-MSC CM injection reduces mouse body weight and eAT tissue mass. In our previous study, we demonstrated that T-MSC CM produces anti-adipogenic effects. In the present study, these in vitro findings were further evaluated in a mouse model of accelerated senescence (SPAM6 mice), as these mice have been reported to exhibit an obese phenotype (26). Control culture medium or T-MSC CM was injected into 7-month-old SAMP6 mice, and body weight changes were examined 2 months later. A significant decrease in the weight of mice was observed at 9 months in mice injected with T-MSC CM, whereas the mice injected with the control medium did not exhibit alterations in weight (Fig. 1A). Isolated organs were weighed and normalized to the body weight. T-MSC CM injection led to reduced eAT mass compared with control mice, whereas the inguinal adipose tissue, liver and muscle mass were not affected by injection of T-MSC CM (Fig. 1B). Histological analysis of eAT demonstrated a reduction in the size of adipocytes in the T-MSC CM-injected mice compared with control mice (Fig. 1C). To determine the mRNA expression of adipogenic markers in eAT adipocytes, collagen digestion was performed, followed by floating adipocyte fraction separation. Together with the reduction in eAT mass and adipocyte size, the mRNA expression of the adipogenic markers peroxisome proliferator-activated receptor $\gamma(\operatorname{PPAR} \gamma)$, CCAAT/enhancer-binding protein $\alpha(\mathrm{C} / \mathrm{EBP} \alpha)$ and leptin were significantly decreased, while adiponectin expression was increased in eAT adipocytes, compared with control-treated mice (Fig. 1D).

T-MSC CM injection increases adiponectin in the circulation by upregulating expression in eAT. As weight reduction is associated with an increase in circulating adiponectin levels, the present study investigated total and HMW adiponectin in mouse plasma prior to ( 7 months) and following (9 months) treatment with T-MSC CM. A significant increase in total adiponectin following T-MSC CM treatment was observed, while levels of adiponectin were significantly decreased in the mice injected with control medium (Fig. 2A). In addition, levels of HMW adiponectin were increased following T-MSC CM injection, although this was not statistically significant (Fig. 2B). Total and HMW adiponectin levels in mouse plasma were normalized to 
Table I. Sequences of primers used for quantitative polymerase chain reaction.

Primer sequence

\begin{tabular}{lcll}
\multirow{2}{*}{ Gene } & GeneBank accession & \multicolumn{1}{c}{ Forward } & \multicolumn{1}{c}{ Reverse } \\
\cline { 3 - 4 } Cyclophilin & NM_021130.4 & 5'-CGTTTTGGGTCCAGGAATGG-3' & 5'-TACAGGACATTGCGAGCAGA-3' \\
PPAR $\gamma$ & NM_011146.3 & 5'-GGAAGACCACTCGCATTCCTT-3' & 5'-GTAATCAGCAACCATTGGGTCA-3' \\
C/EBP $\alpha$ & NM_007678.3 & 5'-CAAGAACAGCAACGAGTACCG-3' & 5'-GTCACTGGTCAACTCCAGCAC-3' \\
Leptin & NM_008493.3 & 5'-GTGGCTTTGGTCCTATCTGTC-3' & 5'-CGTGTGTGAAATGTCATTGATCC-3' \\
Adiponectin & NM_009605.4 & 5'-GTTCCCAATGTACCCATTCGC-3' & 5'-TGTTGCAGTAGAACTTGCCAG-3' \\
p40 ${ }^{\text {phox }}$ & NM_008677.2 & 5'-GCCGCTATCGCCAGTTCTAC-3' & 5'-GCAGGCTCAGGAGGTTCTTC-3' \\
p47phox & NM_010876.4 & 5'-GATGTTCCCCATTGAGGCCG-3' & 5'-GTTTCAGGTCATCAGGCCGC-3' \\
P67phox & NM_010877.5 & 5'-CTGGCTGAGGCCATCAGACT-3' & 5'-AGGCCACTGCAGAGTGCTTG-3' \\
gp91 phox & NM_007807.5 & 5'-TTGGGTCAGCACTGGCTCTG-3' & 5'-TGGCGGTGTGCAGTGCTATC-3'
\end{tabular}

$\operatorname{PPAR} \gamma$, peroxisome proliferator-activated receptor $\gamma$; C/EBP $\alpha$, CCAAT/enhancer-binding protein $\alpha$.

A

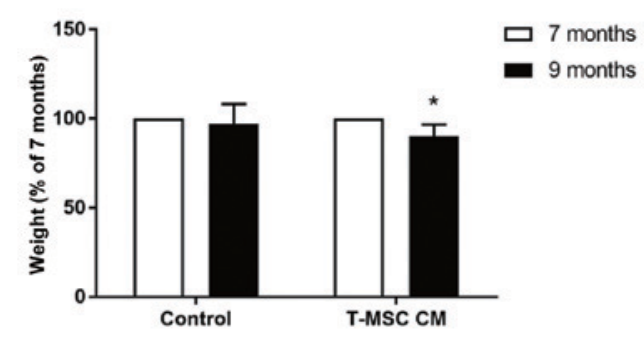

C

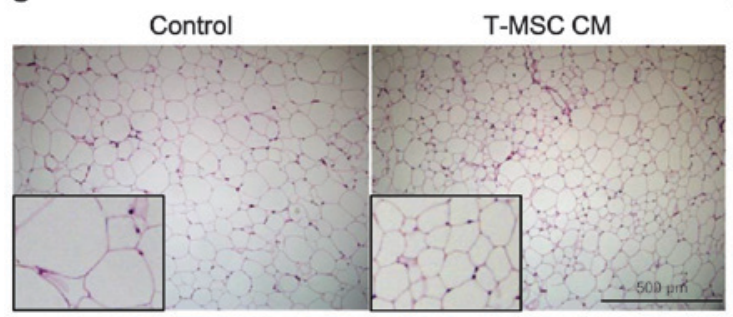

B

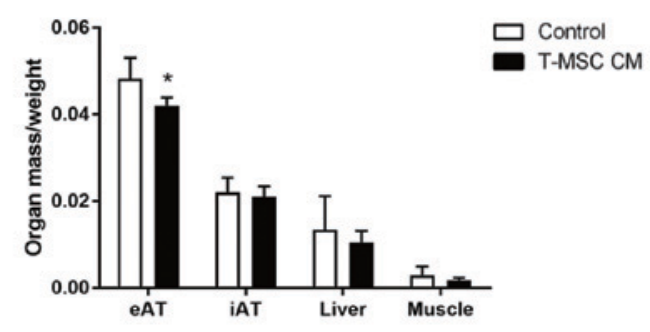

D

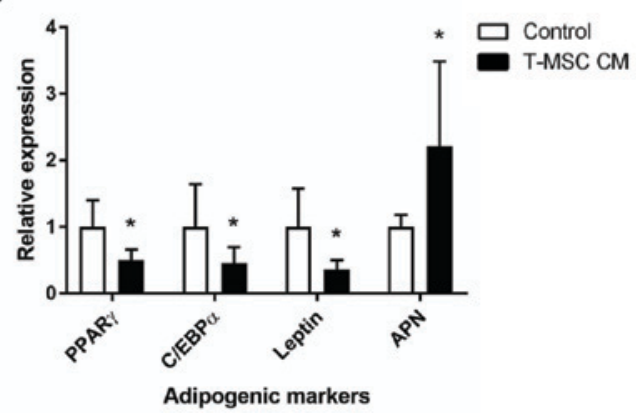

Figure 1. T-MSC CM injection reduced body weight and eAT accumulation in SAMP6 mice. (A) Mouse body weights were measured prior to (7-month-old) and following (9-month-old mice) treatment with control or T-MSC CM. (B) eAT, iAT, liver and skeletal muscle were harvested and weighed upon sacrifice of treated mice. Organ weights were normalized to the body weight of each mouse. (C) Hematoxylin and eosin staining of mouse eAT was performed. Figures are representative of images taken from 10-12 fields of each mouse eAT section. Magnification, x100. Inserts, magnification x250. (D) mRNA expression of the adipogenic genes PPAR $\gamma, \mathrm{C} / \mathrm{EBP} \alpha$, leptin and APN in fractionated eAT adipocytes were assessed by reverse transcription-quantitative polymerase chain reaction. Data are presented as the mean \pm standard error of the mean, $n=6$. ${ }^{*} \mathrm{P}<0.05$ vs. control. T-MSC CM, tonsil-derived mesenchymal stem cell conditioned medium; eAT, epididymal adipose tissue; SAMP6, senescence-accelerated mouse prone 6; iAT, inguinal AT; PPAR $\gamma$, peroxisome proliferator-activated receptor $\gamma ; \mathrm{C} / \mathrm{EBP} \alpha, \mathrm{CCAAT/enhancer-binding} \mathrm{protein} \alpha$; APN, adiponectin.

the eAT mass and the results demonstrated a significant increase in the levels of total and HMW adiponectin following treatment with T-MSC CM, compared with control-treated mice (Fig. 2C). Furthermore, adiponectin protein levels in eAT lysates were significantly increased by T-MSC CM treatment compared with control treatment (Fig. 2D). Non-reduced and non-denatured tissue lysates were used to determine adiponectin multimerization. These experiments indicated that T-MSC CM enhances formation of adiponectin multimers (Fig. 2E). In addition, the present study investigated whether oxidative stress in the adipose tissue, an established negative regulator of adiponectin expression in aging, is modulated in the presence or absence of T-MSC CM injection (27). The mRNA expression of subunits of NADPH oxidase was investigated by RT-qPCR, and the results demonstrated a significant reduction of $\mathrm{p} 40^{\text {phox }}$ and $\mathrm{gp} 91^{\text {phox }}$ in eAT from mice injected with T-MSC CM compared with controls (Fig. 2F).

Adiponectin secretion and multimerization are enhanced by T-MSC CM treatment. The current study confirmed these 
A

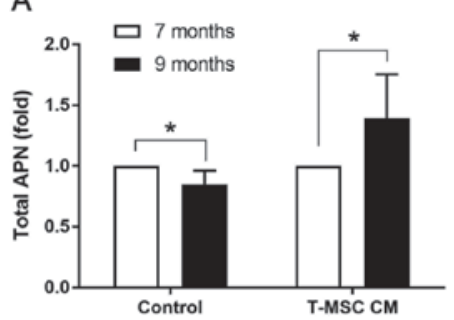

B

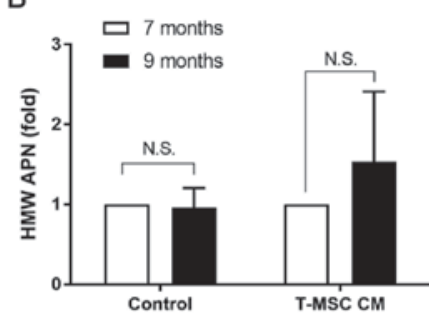

C

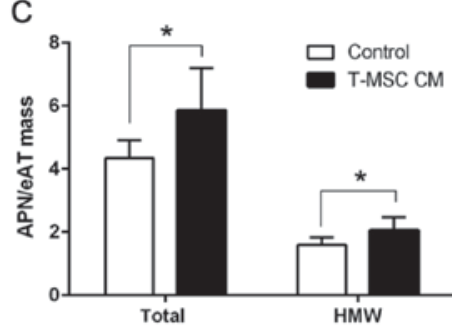

D
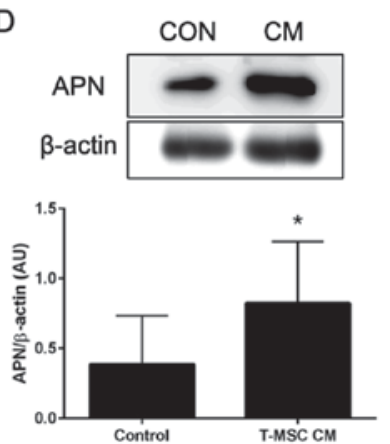

E

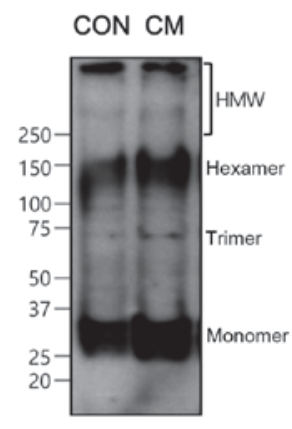

F

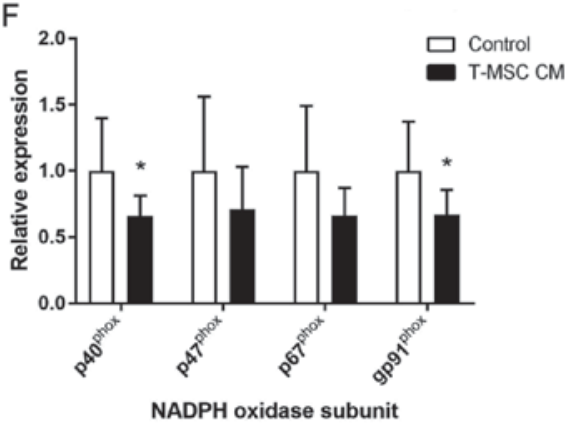

Figure 2. T-MSC CM injection increases APN in the plasma and eAT of SAMP6 mice. (A) Total and (B) HMW APN in mouse plasma prior to (7-month-old) and following (9-month-old) treatment with T-MSC CM or control medium were measured by ELISA. Results were expressed as a fold-change compared to the levels measured in 7-month-old mice. (C) Total and HMW adiponectin in-/+ T-MSC CM-treated mouse plasma was normalized to the eAT mass of each mouse. (D) Adiponectin expression levels in eAT lysates were determined by western blot analysis. (E) Adiponectin multimer composition in eAT lysates was analyzed by SDS-PAGE on gradient gels under non-reducing and non-heat-denaturing conditions. (F) mRNA expression of oxidative stress markers p40 ${ }^{\text {phox }}$, $\mathrm{p} 47^{\text {phox }}, \mathrm{p} 67^{\text {phox }}$ and gp91 ${ }^{\text {phox }}$ in eAT were examined by reverse transcription-quantitative polymerase chain reaction. Blots are representative images and graphs present quantification for 6 mice per group. Data are presented as the mean \pm standard error of the mean, $\mathrm{n}=6$. ${ }^{*} \mathrm{P}<0.05 \mathrm{vs}$. control. T-MSC CM, tonsil-derived mesenchymal stem cell conditioned medium; APN, adiponectin; eAT, epididymal adipose tissue; SAMP6, senescence-accelerated mouse prone 6; HMW, high molecular weight; CON, control.

in vivo findings in vitro by performing similar experiments with the murine adipocyte cell line 3T3-L1. Preadipocytes were induced to differentiate to adipocytes for 7 days and subsequently treated with control medium or T-MSC CM for an additional 3 days. Total RNA or whole cell lysates were collected, and adiponectin expression was examined by RT-qPCR and western blot analysis. No significant changes in the cellular levels of adiponectin mRNA or protein were observed following treatment with T-MSC CM, compared with controls (Fig. 3A and B), however, adiponectin secretion into the culture medium was significantly increased in cells treated with T-MSC CM compared with controls (Fig. 3C). Adiponectin multimer formation was also investigated under non-reduced and non-heat-denatured conditions. T-MSC CM treatment slightly increased the formation of HMW adiponectin by differentiated 3T3-L1 adipocytes (Fig. 3D).

T-MSC CM treatment ameliorates oxidative stress and restores adiponectin multimerization. In order to extend the in vivo findings of the reduction in NADPH oxidase subunit transcription, we induced oxidative stress in mature 3T3-L1 adipocytes using glucose oxidase (28). Cells were differentiated for 10 days, challenged with glucose oxidase for $2 \mathrm{~h}$ and treated with control medium or T-MSC CM for the following $24 \mathrm{~h}$. Cell culture medium and cells were collected and ROS/RNS production was measured. The results demonstrated that enhanced ROS/RNS generation in glucose oxidase-treated cells was significantly ameliorated in cells incubated with T-MSC CM, in culture medium and cells (Fig. 4A and B). Adiponectin expression was also determined, and T-MSC CM treatment was not able to reverse reduced mRNA expression induced by glucose oxidase (Fig. 4C), however, HMW adiponectin formation was restored to a certain extent in cells treated with T-MSC CM (Fig. 4D).

\section{Discussion}

The present study has demonstrated the effects of T-MSC $\mathrm{CM}$ treatment on weight reduction and the promotion of adiponectin secretion from adipocytes using SAMP6 aging mice and 3T3-L1 adipocytes. Obesity increases inflammation and insulin resistance in organs. This pathophysiology is associated with a reduction in the anti-inflammatory adipokine adiponectin the formation of HMW adiponectin in adipocytes. The results of the current study indicated that T-MSC CM infusion in mice reduces eAT accumulation and increases adiponectin synthesis, secretion and multimerization.

T-MSC CM injection resulted in weight loss in mice via a reduction in visceral adiposity and adipocyte size. Investigation of the expression of adipogenic markers in eAT adipocytes revealed downregulation of the transcription factors PPAR $\gamma$ and $\mathrm{C} / \mathrm{EBP} \alpha$ following T-MSC CM injection, and these results are consistent with our previous findings that T-MSC CM treatment inhibits adipocyte differentiation in vitro (14). The reduced expression of adipogenic regulators 
A

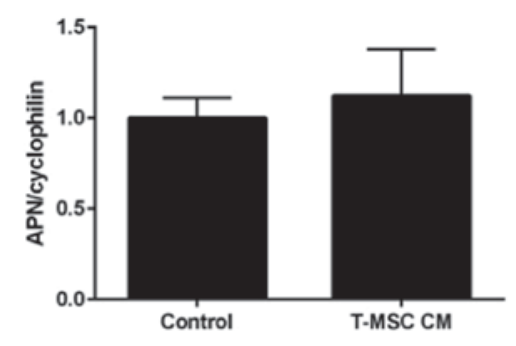

C
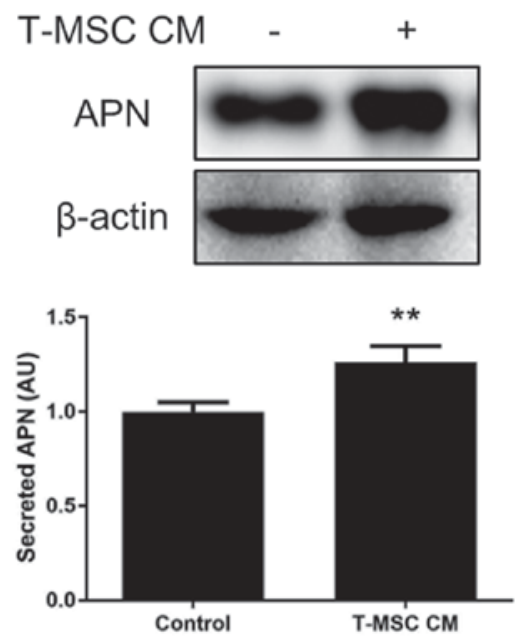

B

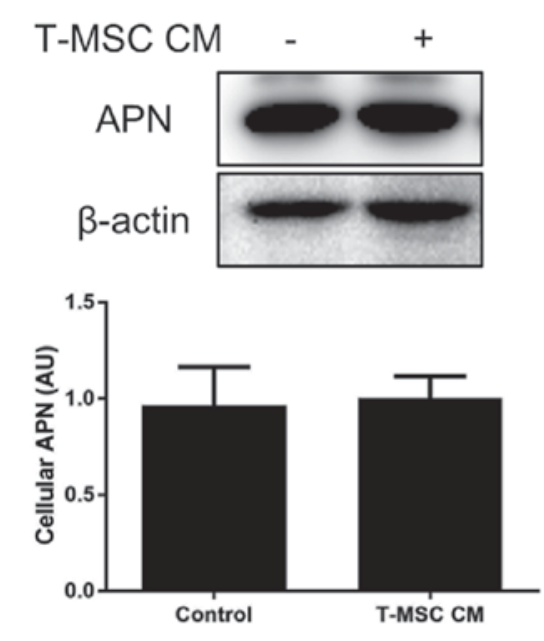

D

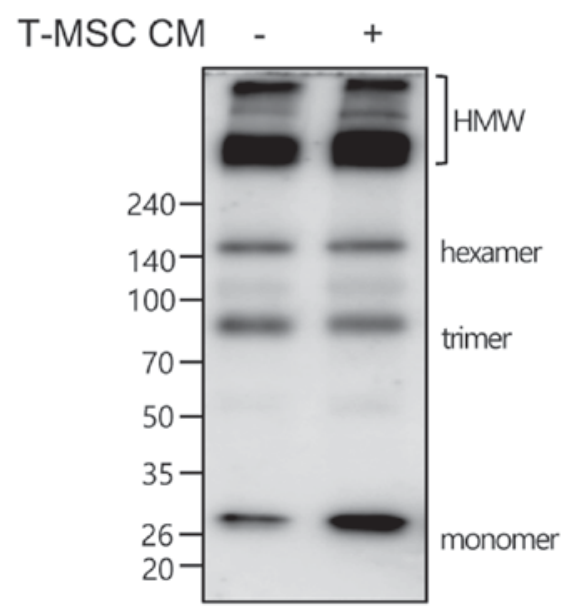

Figure 3. T-MSC CM treatment increases APN secretion in 3T3-L1 adipocytes. 3T3-L1 preadipocytes were induced to differentiate into adipocytes using a standard protocol until differentiation day 7, cells were then incubated with or without T-MSC CM from day 7 to day 10 . Cells were incubated in serum-free medium overnight, and culture supernatants and cells were harvested for further analyses. (A) mRNA expression of APN was measured using reverse transcription-quantitative polymerase chain reaction. APN in (B) total cell lysates and (C) culture supernatants was investigated using western blot analysis. (D) APN multimer composition in culture supernatants was analyzed by SDS-PAGE on gradient gels under non-reducing and non-heat-denaturing conditions. Data are presented as the mean \pm standard error of the mean, $\mathrm{n}=4$. ${ }^{*} \mathrm{P}<0.01 \mathrm{vs}$. control. T-MSC CM, tonsil-derived mesenchymal stem cell conditioned medium; APN, adiponectin; HMW, high molecular weight.

in T-MSC CM-injected mice confirmed the anti-adipogenic effects of T-MSC CM in vivo.

An increase in adiponectin expression was observed in T-MSC CM-treated mice, while PPAR $\gamma$ expression was reduced, which is a master regulator of adipogenesis. Regarding promotion or inhibition of adipogenesis, conflicting results currently exist. The promotion of adipogenesis is required for the generation of small and insulin-sensitive adipocytes. This restrains expansion of adipose tissue via adipocyte hypertrophy, which may lead to insulin resistance and ectopic lipid accumulation (29). On the other hand, it has also been demonstrated that blocking adipocyte differentiation using a PPAR $\gamma$ antagonist protected mice from high fat diet-induced adipocyte hypertrophy and insulin resistance (30). The results of the present study demonstrate that anti-adipogenic effect of T-MSC CM involves reduction of adipocyte size and increased adiponectin synthesis. These effects may yield medical benefits and further studies are required to elucidate whether T-MSC CM may also be able to improve inflammation and insulin sensitivity.

The SAMP6 mouse strain is useful for the study of obesity in aging as reduced physical performance and energy metabolism lead to the development of obesity in this mouse strain. Furthermore, a previous report demonstrated that SAMP6 mice develop characteristics of obesity (26). A significantly higher BMI and the increase of obesity parameters in the plasma, including glucose, triglyceride, insulin and leptin, were reported in SAMP6 mice compared with age-matched AKR/J or SAMR1 mice. In addition, adiponectin in plasma was reported to be significantly lower in SAMP6 mice compared with control mice, and low adiponectin is an established characteristic of obesity $(31,32)$. However, the 
A

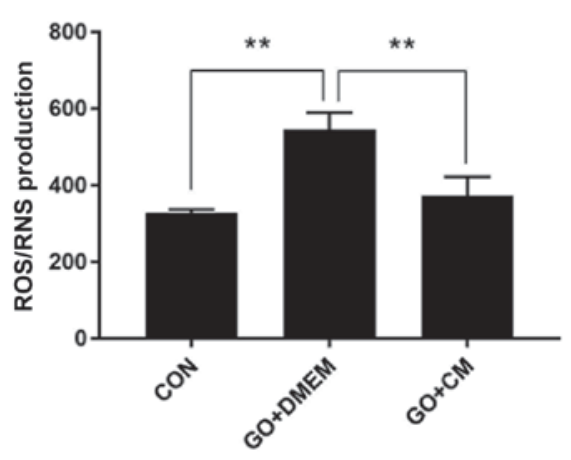

C

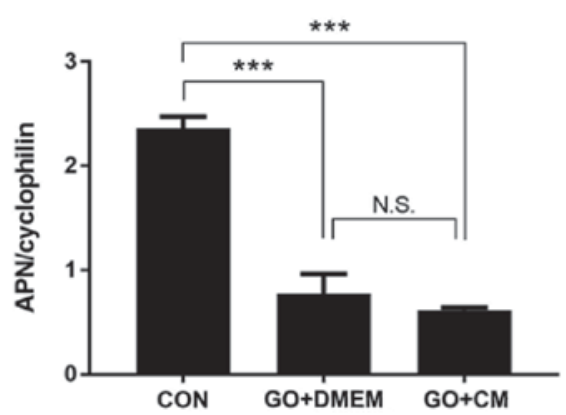

B
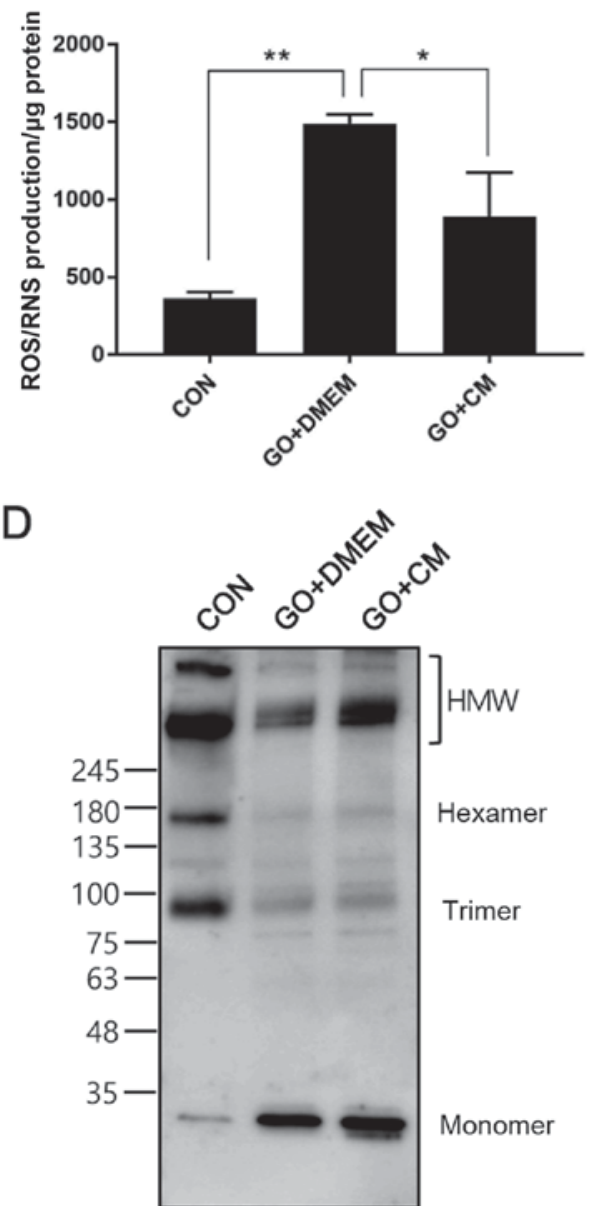

Figure 4. T-MSC CM treatment reduces oxidative stress and restores HMW APN formation in 3T3-L1 adipocytes. 3T3-L1 adipocytes were differentiated for 10 days and challenged with GO for $2 \mathrm{~h}$, followed by incubation in serum-free medium overnight in the presence or absence of T-MSC CM. ROS/RNS production in (A) culture supernatants and (B) cell lysates was measured. (C) mRNA expression of APN was measured using reverse transcription-quantitative polymerase chain reaction. (D) APN multimer composition in culture supernatants was analyzed by SDS-PAGE on gradient gels under non-reducing and non-heat-denaturing conditions. Data are presented as the mean \pm standard error of the mean, $\mathrm{n}=3$. ${ }^{*} \mathrm{P}<0.05,{ }^{* *} \mathrm{P}<0.01$ and ${ }^{* * * *} \mathrm{P}<0.001$, as indicated. T-MSC CM, tonsil-derived mesenchymal stem cell conditioned medium; HMW, high molecular weight; APN, adiponectin; GO, glucose oxidase; ROS, reactive oxygen species; RNS, reactive nitrogen species; CM, T-MSC CM; DMEM, Dulbecco's modified Eagle's medium; CON, control; N.S., not significant.

observations in the present study should be confirmed using a diet-induced obesity mouse model. More detailed analyses on the consequences of adiponectin induction on body metabolism are required. The action of T-MSC CM on other metabolic organs, such as the liver or skeletal muscle, needs to be determined. Identification of adiponectin receptors that are involved in T-MSC CM-induced adiponectin function and changes in the signal transduction may explain the association between T-MSC CM and adiponectin. In addition, the association between increases in adiponectin and improvement in metabolic parameters requires further investigation. Measurement of blood glucose, triglyceride and inflammatory markers, as well as performing glucose tolerance and insulin tolerance tests in the presence or absence of T-MSC CM may increase our understanding of the advantages of using T-MSC $\mathrm{CM}$ in the regulation of metabolic diseases.

Oxidative stress is one of the key contributors of aging pathologies. In obesity, systemic oxidative stress is closely associated with the incidence of metabolic syndrome. One potential mechanism of action is that high levels of ROS production may lead to suppressed adiponectin secretion $(27,33)$. In addition, a recent report demonstrated a positive correlation between plasma adiponectin levels and antioxidant capacity in the elderly (34). The present study elucidated effects of T-MSC CM on the promotion of adiponectin secretion in the setting of both obesity and aging. Furthermore, the results demonstrated that T-MSC CM led to an increase in the HMW adiponectin formation with the amelioration of oxidative stress. Further studies are required to identify potential antioxidant molecules secreted by T-MSCs and their mechanisms of actions.

In conclusion, we demonstrated that T-MSC CM injection reduces mouse body weight and eAT mass. Reduction in body weight increased adiponectin in circulation by upregulating adiponectin expression and multimerization. Amelioration of oxidative stress may be the mechanism by which T-MSC CM leads to increased adiponectin secretion. Furthermore, these results, which, to the best of our knowledge, provide the first description of the role of T-MSC CM on adiponectin synthesis and secretion, may provide the framework for future development of cell therapy to combat obesity or obesity-associated metabolic diseases. 


\section{Acknowledgements}

This study was supported by the Bio \& Medical Technology Development Program of the National Research Foundation of Korea funded by the Ministry of Science, ICT \& Future Planning (grant no. 2012M3A9C6049823), Ministry of Education (grant no. 2016R1A6A3A11933360) and Intramural Research Promotion Grants from Ewha Womans University School of Medicine (Seoul, Korea).

\section{References}

1. Lavoie JR and Rosu-Myles M: Uncovering the secretes of mesenchymal stem cells. Biochimie 95: 2212-2221, 2013.

2. Konala VB, Mamidi MK, Bhonde R, Das AK, Pochampally R and Pal R: The current landscape of the mesenchymal stromal cell secretome: A new paradigm for cell-free regeneration. Cytotherapy 18: 13-24, 2016.

3. Li TS, Takahashi M, Ohshima M, Qin SL, Kubo M, Muramatsu K and Hamano K: Myocardial repair achieved by the intramyocardial implantation of adult cardiomyocytes in combination with bone marrow cells. Cell Transplant 17: 695-703, 2008.

4. Siegel G, Krause P, Wöhrle S, Nowak P, Ayturan M, Kluba T, Brehm BR, Neumeister B, Köhler D, Rosenberger P, et al: Bone marrow-derived human mesenchymal stem cells express cardiomyogenic proteins but do not exhibit functional cardiomyogenic differentiation potential. Stem Cells Dev 21: 2457-2470, 2012.

5. Bi B, Schmitt R, Israilova M, Nishio H and Cantley LG: Stromal cells protect against acute tubular injury via an endocrine effect. J Am Soc Nephrol 18: 2486-2496, 2007.

6. Cantinieaux D, Quertainmont R, Blacher S, Rossi L, Wanet T, Noël A, Brook G, Schoenen J and Franzen R: Conditioned medium from bone marrow-derived mesenchymal stem cells improves recovery after spinal cord injury in rats: An original strategy to avoid cell transplantation. PLoS One 8: e69515, 2013.

7. Janjanin S, Djouad F, Shanti RM, Baksh D, Gollapudi K, Prgomet D, Rackwitz L, Joshi AS and Tuan RS: Human palatine tonsil: A new potential tissue source of multipotent mesenchymal progenitor cells. Arthritis Res Ther 10: R83, 2008.

8. Ryu KH, Cho KA, Park HS, Kim JY, Woo SY, Jo I, Choi YH, Park YM, Jung SC, Chung SM, et al: Tonsil-derived mesenchymal stromal cells: Evaluation of biologic, immunologic and genetic factors for successful banking. Cytotherapy 14: 1193-1202, 2012.

9. Ryu KH, Kim SY, Kim YR, Woo SY, Sung SH, Kim HS, Jung SC, Jo I and Park JW: Tonsil-derived mesenchymal stem cells alleviate concanavalin A-induced acute liver injury. Exp Cell Res 326: 143-154, 2014.

10. Park M, Kim YH, Woo SY, Lee HJ, Yu Y, Kim HS, Park YS, Jo I, Park JW, Jung SC, et al: Tonsil-derived mesenchymal stem cells ameliorate CCl4-induced liver fibrosis in mice via autophagy activation. Sci Rep 5: 8616, 2015.

11. Kim SY, Kim YR, Park WJ, Kim HS, Jung SC, Woo SY, Jo I, Ryu KH and Park JW: Characterisation of insulin-producing cells differentiated from tonsil derived mesenchymal stem cells. Differentiation 90: 27-39, 2015.

12. Park YS, Kim HS, Jin YM, Yu Y, Kim HY, Park HS, Jung SC, Han KH, Park YJ, Ryu KH and Jo I: Differentiated tonsil-derived mesenchymal stem cells embedded in Matrigel restore parathyroid cell functions in rats with parathyroidectomy. Biomaterials 65: 140-152, 2015.

13. Park S, Choi Y, Jung N, Yu Y, Ryu KH, Kim HS, Jo I, Choi BO and Jung SC: Myogenic differentiation potential of human tonsil-derived mesenchymal stem cells and their potential for use to promote skeletal muscle regeneration. Int J Mol Med 37: 1209-1220, 2016.

14. Kim YH, Park M, Cho KA, Kim BK, Ryu JH, Woo SY and Ryu KH: Tonsil-derived mesenchymal stem cells promote bone mineralization and reduce marrow and visceral adiposity in a mouse model of senile osteoporosis. Stem Cells Dev 25: 1161-1171, 2016.

15. Ryu JH, Park M, Kim BK, Ryu KH and Woo SY: Tonsil-derived mesenchymal stromal cells produce CXCR2-binding chemokines and acquire follicular dendritic cell-like phenotypes under TLR3 stimulation. Cytokine 73: 225-235, 2015.
16. Park M, Kim YH, Ryu JH, Woo SY and Ryu KH: Immune suppressive effects of tonsil-derived mesenchymal stem cells on mouse bone-marrow-derived dendritic cells. Stem Cells Int 2015: 106540, 2015.

17. Cho KA, Lee JK, Kim YH, Park M, Woo SY and Ryu KH: Mesenchymal stem cells ameliorate B-cell-mediated immune responses and increase IL-10-expressing regulatory B cells in an EBI3-dependent manner. Cell Mol Immunol: Jan 2, 2017 (Epub ahead of print).

18. Kim JY, Park M, Kim YH, Ryu KH, Lee KH, Cho KA and Woo SY: Tonsil-derived mesenchymal stem cells (T-MSCs) prevent Th17-mediated autoimmune response via regulation of the programmed death-1/programmed death ligand-1 (PD-1/PD-L1) pathway. J Tissue Eng Regen Med: Jan 20, 2017 (Epub ahead of print).

19. Whitehead JP, Richards AA, Hickman IJ, Macdonald GA and Prins JB: Adiponectin-a key adipokine in the metabolic syndrome. Diabetes Obes Metab 8: 264-280, 2006.

20. Liu M and Liu F: Regulation of adiponectin multimerization, signaling and function. Best Pract Res Clin Endocrinol Metab 28: 25-31, 2014

21. Wang ZV and Scherer PE: Adiponectin, the past two decades. J Mol Cell Biol 8: 93-100, 2016.

22. Cnop M, Havel PJ, Utzschneider KM, Carr DB, Sinha MK, Boyko EJ, Retzlaff BM, Knopp RH, Brunzell JD and Kahn SE: Relationship of adiponectin to body fat distribution, insulin sensitivity and plasma lipoproteins: Evidence for independent roles of age and sex. Diabetologia 46: 459-469, 2003.

23. Furler SM, Gan SK, Poynten AM, Chisholm DJ, Campbell LV and Kriketos AD: Relationship of adiponectin with insulin sensitivity in humans, independent of lipid availability. Obesity (Silver Spring) 14: 228-234, 2006.

24. Yu Y, Park YS, Kim HS, Kim HY, Jin YM, Jung SC, Ryu KH and Jo I: Characterization of long-term in vitro culture-related alterations of human tonsil-derived mesenchymal stem cells: Role for CCN1 in replicative senescence-associated increase in osteogenic differentiation. J Anat 225: 510-518, 2014.

25. Livak KJ and Schmittgen TD: Analysis of relative gene expression data using real-time quantitative PCR and the 2(-Delta Delta C(T)) Method. Methods 25: 402-408, 2001.

26. Niimi K, Takahashi E and Itakura C: Adiposity-related biochemical phenotype in senescence-accelerated mouse prone 6 (SAMP6). Comp Med 59: 431-436, 2009.

27. Furukawa S, Fujita T, Shimabukuro M, Iwaki M, Yamada Y, Nakajima Y, Nakayama O, Makishima M, Matsuda M and Shimomura I: Increased oxidative stress in obesity and its impact on metabolic syndrome. J Clin Invest 114: 1752-1761, 2004.

28. Guo H, Ling W, Wang Q, Liu C, Hu Y and Xia M: Cyanidin 3-glucoside protects 3T3-L1 adipocytes against $\mathrm{H} 2 \mathrm{O} 2$ - or TNF-alpha-induced insulin resistance by inhibiting c-Jun NH2-terminal kinase activation. Biochem Pharmacol 75: 1393-1401, 2008

29. Gustafson B, Hammarstedt A, Hedjazifar S and Smith U: Restricted adipogenesis in hypertrophic obesity: The role of WISP2, WNT, and BMP4. Diabetes 62: 2997-3004, 2013.

30. Choi SS, Kim ES, Jung JE, Marciano DP, Jo A, Koo JY, Choi SY, Yang YR, Jang HJ, Kim EK, et al: PPAR $\gamma$ antagonist Gleevec improves insulin sensitivity and promotes the browning of white adipose tissue. Diabetes 65: 829-839, 2016.

31. Matsubara M, Maruoka S and Katayose S: Inverse relationship between plasma adiponectin and leptin concentrations in normal-weight and obese women. Eur J Endocrinol 147: 173-180, 2002.

32. Asayama K, Hayashibe H, Dobashi K, Uchida N, Nakane T, Kodera K, Shirahata A and Taniyama M: Decrease in serum adiponectin level due to obesity and visceral fat accumulation in children. Obes Res 11: 1072-1079, 2003.

33. Fujita K, Nishizawa H, Funahashi T, Shimomura I and Shimabukuro M: Systemic oxidative stress is associated with visceral fat accumulation and the metabolic syndrome. Circ J 70: $1437-1442,2006$

34. Gradinaru D, Margina D, Borsa C, Ionescu C, Ilie M, Costache M, Dinischiotu A and Prada GI: Adiponectin: Possible link between metabolic stress and oxidative stress in the elderly. Aging Clin Exp Res: Sep 29, 2016 (Epub ahead of print). 